\title{
Percepção de Saúde e Satisfação com a Vida em Adolescentes: Diferença entre os Sexos
}

\author{
Health Perception and Satisfaction With Life in Adolescents: Difference Between the \\ Sexes
}

Percepción de Salud y Satisfacción con la Vida en Adolescentes: Diferencias entre los Sexos

Miriam Raquel Wachholz Strelhow ${ }^{I}$

Cheila de Oliveira Bueno

Universidade Luterana do Brasil/Canoas

Sheila Gonçalves Câmara

Universidade Luterana do Brasil/Canoas

Universidade Federal de Ciências da Saúde de Porto Alegre

\begin{abstract}
Resumo
O presente estudo avaliou a diferença entre meninos e meninas adolescentes em termos de percepção de saúde, felicidade e satisfação com a vida. A partir das diferenças encontradas, foi avaliada a relação entre a satisfação com diferentes domínios da vida e as variáveis relacionadas à percepção de saúde. A amostra constituiu-se de 188 alunos de oitava série do ensino fundamental das escolas estaduais de Cachoeirinha/RS. Como instrumentos utilizaram-se o Comportamentos de Saúde entre Escolares e a Escala Multidimensional Breve de Satisfação com a vida entre Estudantes. Os dados foram analisados através de estatística univariada e bivariada. Resultados revelam que os meninos apresentam percepção de saúde significativamente mais positiva enquanto as meninas apresentam com maior freqüência emoções negativas. A percepção de saúde relaciona-se positivamente com a satisfação com a experiência como estudante, consigo mesmo e com a vida em geral, já as emoções negativas associam-se inversamente à satisfação nos domínios estudados. Os resultados revelam a importância de ampliar a concepção de saúde nesta população para a promoção da saúde adolescente.
\end{abstract}

Palavras-chave: adolescência; saúde; satisfação com a vida; diferença entre sexos.

\begin{abstract}
The present study assessed the difference between adolescent boys and girls in terms of their perception of health, happiness and satisfaction with their own lives. Bearing in mind the differences identified, an assessment was made of the relationship between their satisfaction with different aspects of their lives, on the one hand, and variables related to their perception of health, on the other. The sample consisted of 188 students from the eighth grade of the basic education of six state public schools of Cachoeirinha/RS. The instruments used were the Health Behavior in Schoolchildren and Brief Life Satisfaction Scale (BLSS). The data were analyzed through univariate and bivariate analysis. Results show that boys present significantly superior health perception, while girls present more often negative emotions. The health perception is positively related to satisfaction with their experience as students, with themselves and with life in general, while negative emotions are inversely related to satisfaction in the studied domains. The results show the importance of broadening the conception of health in this population in order to promote adolescents' health.
\end{abstract}

Keywords: Adolescence; health; satisfaction with life; difference between sexes

\section{Resumen}

El presente estudio evaluó la diferencia entre chicos y chicas adolescentes en términos de percepción de salud, felicidad y satisfacción con la vida. A partir de las diferencias encontradas fue evaluada la relación entre la satisfacción con diferentes dominios de la vida y las variables relacionadas a la percepción de salud. La muestra se compuso por 188 alumnos de octavo año de la enseñanza básica de escuelas estaduales de Cachoeirinha/RS. Los instrumentos utilizados fueron el Comportamientos de Salud entre Estudiantes y la Escala Multidimensional Breve de Satisfacción con la vida entre Estudiantes. Los datos fueron analizados a través de estadística univariada y bivariada. Resultados revelan que los chicos presentan percepción de salud significativamente más positiva mientras las chicas presentan con mayor frecuencia emociones negativas. La percepción de salud relacionase positivamente con la satisfacción como estudiante, con uno mismo y con la vida en general. Las emociones negativas sin embargo, asocian se inversamente a la satisfacción en los dominios estudiados. Los resultados revelan la importancia de ampliar la concepción de salud en esa populación para la promoción de la salud adolescente.

Palabras-clave: adolescencia; salud; satisfacción con la vida; diferencia entre los sexos

\section{Introdução}

1 Endereço: Rua Valença, 182 - Vila Ipiranga. Porto Alegre, Rio Grande do Sul. CEP: $91370-300$

Tel: (51) 3347-1305 / (51) 9335-9447
Estudos demonstram que os adolescentes se percebem e relatam a adolescência a partir de 
conceitos socialmente instituídos, como um período natural de transição, de crise, de rebeldia e conflitos (Ozella \& Aguiar, 2008). Neste estudo, entende-se a adolescência não como um fenômeno universal, igualitário para todos os sujeitos, mas sim, como uma categoria sociocultural, historicamente construída a partir de critérios múltiplos que abrangem tanto a dimensão bio-psicológica, quanto à cronológica e a social. Estar na adolescência é viver uma fase em que múltiplas mudanças acontecem e se refletem no corpo físico, bem como no aspecto psicológico (Ferreira, Alvinir, Teixeira \& Veloso, 2007).

Assim, esta fase não deve ser tomada como um conjunto de fenômenos universais implicados no crescimento e desenvolvimento físico-mental, pois as transformações pelas quais passam os adolescentes também resultam de processos inerentes aos contextos sociais (históricos, políticos e econômicos) nos quais os sujeitos estão imersos. Ozella e Aguiar (2008) afirmam que a concepção de adolescência é construída a partir da realidade objetiva e concreta, por isso fatores como classe social, raça, sexo, idade e cultura são importantes na construção do sentido de ser adolescente.

Os autores destacam a necessidade de cuidado em não apontar o ambiente como único determinante das características dos adolescentes, mas sim como um propiciador, em que cada adolescente irá constituirse a partir de suas vivências, atividades e contexto. A partir desta visão da adolescência ser constituída por múltiplos fatores, pensar a saúde do adolescente implica pensar nos diversos modos de viver a adolescência e de viver a vida.

Através de uma compreensão do comportamento de saúde como resultado da interação entre pessoas e entre pessoas e ambientes, destaca-se a importância do contexto social onde ocorrem tais interações (Rodríguez Marín, Martínez García \& Valcárcel, 1990; Rodríguez Marín \& García, 1995). Em se tratando de crianças e adolescentes, é nesse contexto específico de pertença que se desenvolvem todas as relações que passarão a constituir a identidade e os modos de atuação do sujeito. Ali se dão as primeiras aprendizagens, juízos e valores que este realiza acerca de si mesmo e de seu ambiente, constituindo-se em sua referência em âmbito geral e relacionado à saúde. Para Rodriguez-Marin e García (1995) o contexto social, no qual a criança ou adolescente está inserido, adquire especial importância quando se considera suas propriedades interativas, através das quais se aprendem, desenvolvem, mantêm e emitem os comportamentos orientados para a saúde.

Conforme Huici (1985), as definições acerca do que é considerado saúde, são dadas por este mesmo contexto, da mesma forma que são avaliadas as condutas como adequadas ou inadequadas. Assim, como o ambiente social consiste em um importante referencial em termos de apoio, também é o responsável, muitas vezes, pela estigmatização e discriminação dos sujeitos de acordo com seu status frente ao tema saúde-doença. A influência do grupo na interpretação de saúde manifesta-se através das crenças e informações que dispõe, de maneira que as atribuições referentes à enfermidade são conferidas pelo grupo, a ponto mesmo de culpabilizar ou discriminar um indivíduo de acordo com essas diretrizes. Também os hábitos saudáveis e os comportamentos orientados para a saúde originam-se no cerne do grupo, de maneira que este tem importante papel na promoção de condutas saudáveis.

Atualmente os estudos referentes ao tema da saúde do adolescente abordam principalmente temas referentes a comportamentos denominados "de risco" (abuso de drogas, gravidez e DST's, e violência) como possíveis determinantes para a definição de saúde desta população (Ozella \& Aguiar, 2008). Tais fatores são relevantes à medida que são identificados como práticas exercidas a partir das experiências vivenciadas no contexto onde o adolescente está inserido. Isso demonstra que na adolescência, a saúde é possivelmente determinada pelas condições que o contexto social (relações familiares, sociais e escolares) oferece.

No entanto, é preciso ampliar a concepção de saúde na adolescência, definindo-a não apenas pela ausência de agravos ou comportamentos que implicam em risco para a saúde, mas definindo-a como qualidade de vida. De acordo com Kuczynski (2010), ao falar em qualidade de vida na infância e adolescência, a prioridade deve ser formular conceitos que realmente traduzam os interesses desse público, e avaliar de forma a captar suas percepções e não as dos adultos a sua volta. Por isso faz-se importante investigar a percepção que os próprios adolescentes têm de sua saúde.

Costa e Bigras (2007) apontam que a questão de saúde e bem-estar na adolescência, assim como na infância, deve ter um enfoque diferenciado em relação à população adulta, e as ações devem envolver aspectos do macro e micro ambientes, considerando os determinantes sociais (sistemas de saúde, educação, trabalho, desenvolvimento social, direitos, necessários à integração social das famílias) - espaços de convivência, formação e intervenção; bem como os determinantes familiares (influenciados pelo ambiente sociocultural), além de aspectos pessoais-individuais.

No estudo de Garbin, Garbin, Moimaz e Gonçalves (2009) com adolescentes, 34,5\% conceituaram saúde como coisa ou algo que precisam cuidar; $26,6 \%$, como bem-estar; $18,1 \%$, como a própria existência; e 14,5\%, como ausência de doença. Já Cromack, Bursztyn e Tura, (2009), avaliando as representações sociais de saúde de adolescentes com idades de 14 a 18 anos, identificaram que entre os diferentes significados atribuídos a saúde pelos adolescentes, 
foram apontados elementos como paz, amor, saudável, carinho, alegria, vida, atividades físicas, lazer, alimentação, demonstrando que para eles a saúde também é interpretada com uma conotação positiva.

A satisfação com a vida é considerada como a medida positiva que a pessoa faz da sua vida em geral, bem como de aspectos específicos, como família, trabalho, estudo, lazer, amigos e saúde, constituindo-se em um componente cognitivo do bem-estar subjetivo, (Diener, 1994; García, 2002; García-Viniegras \& González, 2000; Albuquerque \& Tróccoli, 2004).

De acordo com diferentes autores (Albuquerque \& Tróccoli, 2004; García-Viniegras \& González, 2000; Veenhoven, 1994), este construto seria o resultado da diferença percebida entre as expectativas da pessoa e suas reais conquistas. Pode-se compreendê-lo como um estado psicológico resultante da troca entre o indivíduo e seu entorno micro (família, apoio social) e macrossocial (cultura) (García-Viniegras \& González, 2000).

Assim, o estudo da satisfação com a vida, refere-se a um campo de investigação mais amplo, que engloba aspectos da qualidade de vida das pessoas, estando vinculada à Psicologia Positiva. Os psicólogos ligados a esta linha procuram promover o bem-estar adolescente com o estudo dos fatores que reforçam a satisfação da vida e as variáveis relacionadas (Ma \& Hubner, 2008). Neste sentido, pesquisas como a de Diaz e Alvarado (2007), afirmam existir relação entre satisfação com a vida e aspectos importantes como felicidade e percepção de saúde. Um importante componente da Psicologia Positiva é o bem-estar subjetivo, também chamado de felicidade, pois é um aspecto que pode favorecer a maneira como as pessoas se vêem e como vêem aos outros, podendo resultar em maior prazer na vivência das situações cotidianas e no relacionamento com os pares (Passareli \& Silva, 2007).

Este artigo se propôs a avaliar as diferenças entre meninos e meninas, adolescentes escolares, no que tange a percepção de saúde (avaliação subjetiva e experiência de estados psicológicos - emoções - negativos), percepção de felicidade (avaliação subjetiva) e satisfação dos jovens em relação a diferentes áreas de sua vida (família, amigos, escola, tempo livre, lugar onde vive, consigo mesmo, com sua imagem corporal e com a vida em geral). Como um objetivo adicional, buscou-se avaliar a relação da satisfação com a vida com percepção de saúde, incluindo a experiência de estados psicológicos (emoções) negativos.

\section{Método}

Neste estudo foi utilizado delineamento transversal, com uma amostra de 188 alunos, com idades entre 12 e 19 anos, de oitava série do ensino fundamental das escolas estaduais de Cachoeirinha/ RS, em 2008. O município contava com 13 escolas estaduais que ofereciam este nível de ensino, onde estavam matriculados 1093 alunos em 2008, de acordo com os dados disponibilizados no site da Secretaria da Educação do Rio Grande do Sul (2008). A seleção dos estudantes foi feita através do sorteio de sete escolas, perfazendo a metade de escolas do município que preenchiam os critérios de serem estaduais e oferecerem oitava série do ensino fundamental. Com o sorteio foi possível estabelecer aleatoriedade na distribuição das escolas por regiões. Em cada escola foi sorteada uma turma de oitava série. Considerando-se que as turmas tinham uma média de 30 alunos, a amostra foi prevista em 210 alunos. Os critérios de inclusão dos alunos foram estar na faixa etária entre 12 e 19 anos, em ensino regular no turno diurno e terem trazido o Termo de Consentimento Livre e Esclarecido (TCLE) assinado por pais ou responsáveis, no caso dos menores de 18 anos, ou terem os próprios participantes assinado o TCLE, quando maiores de idade. Dado o número real de alunos matriculados que estavam freqüentando regularmente a escola, de acordo com as listas de presenças dos professores, e os critérios de inclusão, o número de sujeitos na amostra final foi de 188 , revelando uma perda de $10,5 \%$ em relação à amostra planejada.

$\mathrm{O}$ instrumento utilizado consta das seguintes áreas:

1) Percepção de saúde e percepção de felicidade. Foi utilizado o Comportamentos de Saúde entre Escolares (Health Behavior in Schoolchildren), desenvolvido pela OMS para estudar os estilos de vida dos adolescentes em diferentes países (Wold, 1995). O questionário aborda: atividade física, alimentação, consumo de drogas legais e ilegais, higiene dental, hábitos de descanso, respeito pelas normas de trânsito, escola e tempo livre, e percepção de saúde e felicidade. Para o presente estudo foram utilizadas as questões referentes a percepção de saúde, freqüência de sentir-se deprimido/a, irritado/a ou bravo/a e nervoso/a e percepção de felicidade (caracterizando aspectos relacionados à saúde psicológica e sua percepção subjetiva).

A percepção de saúde é avaliada em um bloco de questões que englobam: o quanto o jovem acredita estar saudável em um continuum que vai de muito saudável a nada saudável, pontuado em escala Likert de quatro pontos (1- nada saudável, 2- não muito saudável, 3- bastante saudável e 4- muito saudável); as experiências psicológicas de sentir-se deprimido/a, irritado/a ou bravo/a e nervoso/a, avaliadas em termos da freqüência com que foram experimentadas pelos jovens em escala Likert de cinco pontos (1- quase nunca ou nunca, 2- quase todos os meses, 3- quase todas as semanas, 4- mais de uma vez por semana e 
5- quase todos os dias); e a percepção de felicidade, avaliada em uma pergunta sobre como, de maneira geral, o jovem se sente em sua vida atual (1- não sou nada feliz, 2- não me sinto muito feliz, 3- me sinto bem e 4 - me sinto muito feliz). No presente estudo, a consistência interna entre os cinco itens avaliados (considerados como uma escala) foi de 0,70.

2) Satisfação com a vida. Para avaliar a satisfação com a vida foi utilizada a Escala Multidimensional Breve de Satisfação com a vida entre Estudantes (Brief Multidimensional Students' Life Satisfaction

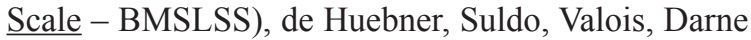
e Zullig (2004). Consiste em uma medida de cinco itens (satisfação com a família, amigos, experiência como estudante, consigo mesmo e com o lugar onde vive), acrescida de um item sobre a satisfação com a vida em geral, a serem respondidos em escala Likert de 11 pontos, com opções que variam de "péssima" a "excelente". A soma dos resultados dos sujeitos nos seis itens fornece um escore de satisfação geral com a vida. No presente estudo, a escala obteve um índice alfa de Cronbach de 0,92 com os seis itens e 0,90 com os cinco itens referentes a domínios específicos.

A coleta de dados foi feita de forma grupal em salas de aula pelas pesquisadoras, com tempo médio de 30 minutos, após autorização das escolas selecionadas. Os participantes responderam ao instrumento de pesquisa mediante autorização prévia (assinatura do Termo de Consentimento Livre e Esclarecido) de seus pais ou responsáveis ou sua (no caso dos maiores de idade). O projeto de pesquisa foi avaliado pelo Comitê de Ética em Pesquisa da instituição dos autores.

Para a análise dos dados foram realizadas: análise descritiva para caracterização da amostra e apresentação dos resultados dos adolescentes em termos de percepção de saúde, felicidade e satisfação com a vida; teste $\underline{\mathrm{t}}$ de $\underline{\text { student }}$ para amostras independentes para avaliar as diferenças entre meninos e meninas no que tange a percepção de saúde, percepção de felicidade, satisfação e estados emocionais negativos (sentir-se deprimido/a, irritado/a ou bravo/a e nervoso/a); e, correlação bivariada de Pearson para avaliar a relação entre satisfação e estados emocionais negativos, percepção de felicidade e de saúde.

\section{Resultados}

Dentre os 188 adolescentes escolares que participaram do estudo, $58 \%$ eram do sexo feminino. A idade média foi de 14,5 anos $(\mathrm{DP}=1,11)$, considerando-se a faixa etária de 12 a 19 anos.

A percepção dos estudantes sobre sua saúde revelou que $52,7 \%$ perceberam-se como bastante saudáveis, 30,3\% como não muito saudáveis, $14,4 \%$ como muito saudáveis e $2,7 \%$ como nada saudáveis. Além dessa percepção geral, no que se refere à experiência de estados psicológicos (emoções) negativos, verificou-se que $110(58,5 \%)$ jovens nunca ou quase nunca se sentem deprimidos, 77 (41\%) nunca ou quase nunca se sentem nervosos e $59(31,4 \%)$ nunca ou quase nunca se sentem irritados ou bravos. Entre os jovens que experimentaram tais emoções, variando de 1 (mais de uma vez ao mês) a 4 (quase todos os dias), sentir-se deprimido apareceu com freqüência quase semanal $(\mathrm{m}=2,11 ; \mathrm{DP}=1,17)$, sentir-se nervoso $(\mathrm{m}=2,58 ; \mathrm{DP}=1,12)$ e irritado ou bravo $(\mathrm{m}=2,63 ; \mathrm{DP}=1,16)$ tiveram freqüência de mais de uma vez por semana.

Quanto à percepção dos adolescentes em relação a sua felicidade, as respostas apontaram para uma avaliação positiva. A maioria $(89,2 \%)$ referiu sentirse feliz ou muito feliz, enquanto $10,2 \%$ relataram não se sentirem muito felizes, e apenas $0,5 \%$ referiu não ser nada feliz.

A satisfação com os diferentes domínios da vida e com a vida em geral também apareceu com médias elevadas (considerando-se que a escala variava de zero - péssima a 10 - excelente, com ponto central de cinco). A média mais elevada revelou uma avaliação muito boa com relação à satisfação com a vida em geral $(m=8,52 ; D P=2,31)$. A satisfação com os amigos $(\mathrm{m}=8,45 ; \quad \mathrm{DP}=2,19)$, consigo mesmo $(\mathrm{m}=8,13$; $\mathrm{DP}=2,60)$, com o lugar onde vive $(\mathrm{m}=8,12 ; \mathrm{DP}=2,62)$ e com a família $(m=7,94 ; \mathrm{DP}=2,73)$ foi descrita como boa. A satisfação com a experiência de estudante obteve a menor média $(\mathrm{m}=7,41 ; \mathrm{DP}=2,87)$, indicando uma avaliação relativamente boa.

$\mathrm{Na}$ comparação entre os sexos, houve diferença significativa entre as médias $(t=-2,91 ; p=0,00)$. Os meninos apresentaram percepção mais positiva de sua saúde $(\mathrm{m}=2,96 ; \mathrm{DP}=0,72)$, em relação às meninas $(\mathrm{m}=2,66 ; \mathrm{DP}=0,68)$. Houve diferença significativa também em relação às médias de deprimido $(\mathrm{t}=3,43$; $\mathrm{p}=0,00)$, irritado ou bravo $(\mathrm{t}=3,07 ; \mathrm{p}=0,00)$ e nervoso $(\mathrm{t}=2,60 ; \mathrm{p}=0,01)$, porém, nestas variáveis as meninas obtiveram médias mais elevadas que os meninos (tabela 1).

As médias de ambos os sexos em relação à percepção de felicidade não apresentaram diferença significativa $(\mathrm{t}=-1,40 ; \mathrm{p}=0,16)$. Da mesma forma, não houve diferença significativa entre as médias de meninas e meninos em termos da satisfação com os amigos $(\mathrm{t}=1,83 ; \mathrm{p}=0,07)$, experiência como estudante $(\mathrm{t}=0,84 ; \mathrm{p}=0,40)$, consigo mesmo $(\mathrm{t}=0,45 ; \mathrm{p}=0,65)$, com o lugar onde vive $(\mathrm{t}=-0,06 ; \mathrm{p}=0,95)$ e com a vida em geral $(t=1,04 ; p=0,30)$, embora as meninas tenham apresentado médias mais elevadas em cinco dos seis domínios avaliados, apenas não no item de satisfação com o lugar em que vivem (tabela 1).

Embora a satisfação com a vida não tenha apresentado médias significativamente diferentes entre os sexos, buscou-se avaliar como a satisfação com a vida relaciona-se com as demais variáveis estudadas a fim de compreender, especialmente, que 
Tabela 1.

Diferenças entre os sexos quanto a percepção de saúde, experiência de estados psicológicos (emoções) negativos, percepção de felicidade e satisfação com a vida em escolares da rede estadual de Cachoeirinha, Cachoeirinha, RS, 2008.

\begin{tabular}{|c|c|c|c|c|c|}
\hline Variáveis & Sexo & Média & $D P$ & $t$ & $p$ \\
\hline \multirow{2}{*}{ Percepção de saúde } & Feminino & 2,66 & 0,68 & \multirow{2}{*}{$-2,91$} & \multirow{2}{*}{0,00} \\
\hline & Masculino & 2,96 & 0,72 & & \\
\hline \multirow{2}{*}{ Sentir-se deprimido/a } & Feminino & 2,17 & 1,39 & \multirow{2}{*}{3,43} & \multirow{2}{*}{0,00} \\
\hline & Masculino & 1,53 & 1,04 & & \\
\hline \multirow{2}{*}{ Sentir-se irritado/a ou bravo/a } & Feminino & 3,11 & 1,49 & \multirow{2}{*}{3,07} & \multirow{2}{*}{0,00} \\
\hline & Masculino & 2,42 & 1,56 & & \\
\hline \multirow{2}{*}{ Sentir-se nervoso/a } & Feminino & 2,80 & 1,54 & \multirow{2}{*}{2,60} & \multirow{2}{*}{0,01} \\
\hline & Masculino & 2,22 & 1,47 & & \\
\hline \multirow{2}{*}{ Percepção de felicidade } & Feminino & 3,31 & 0,72 & \multirow{2}{*}{$-1,40$} & \multirow{2}{*}{0,16} \\
\hline & Masculino & 3,45 & 0,64 & & \\
\hline \multirow{2}{*}{ Satisfação com amigos } & Feminino & 8,71 & 1,91 & \multirow{2}{*}{1,83} & \multirow{2}{*}{0,07} \\
\hline & Masculino & 8,10 & 2,51 & & \\
\hline \multirow{2}{*}{$\begin{array}{l}\text { Satisfação com a experiência de } \\
\text { estudante }\end{array}$} & Feminino & 7,57 & 2,60 & \multirow{2}{*}{0,84} & \multirow{2}{*}{0,40} \\
\hline & Masculino & 7,20 & 3,21 & & \\
\hline \multirow{2}{*}{ Satisfação consigo mesmo } & Feminino & 8,21 & 2,41 & \multirow{2}{*}{0,45} & \multirow{2}{*}{0,65} \\
\hline & Masculino & 8,03 & 2,85 & & \\
\hline \multirow{2}{*}{ Satisfação com o lugar em que vive } & Feminino & 8,11 & 2,55 & \multirow{2}{*}{$-0,06$} & \multirow{2}{*}{0,95} \\
\hline & Masculino & 8,13 & 2,73 & & \\
\hline \multirow{2}{*}{ Satisfação com sua vida em geral } & Feminino & 8,69 & 2,02 & \multirow{2}{*}{1,04} & \multirow{2}{*}{0,30} \\
\hline & Masculino & 8,32 & 2,66 & & \\
\hline
\end{tabular}

aspectos da satisfação relacionam-se à percepção de saúde (atuando como fatores de proteção para os meninos), e à experiência de sentir-se deprimido, nervoso e irritado ou bravo (atuando como fatores de risco para as meninas) (tabela 2).

Verificou-se que uma percepção mais positiva de saúde relacionou-se a maior satisfação com a experiência como estudante, consigo mesmo e com a vida em geral. A maior freqüência em sentir-se deprimido, nervoso, irritado ou bravo associou-se a menor satisfação com todos os domínios estudados. Apenas sentir-se irritado ou bravo não se associou com a satisfação com a vida em geral (tabela 2).

\section{Discussão}

Os resultados demonstraram um cenário positivo e saudável em termos da percepção que os adolescentes escolares têm de sua vida. Essa descrição positiva da vida, também é apontada por Silva et al (2007), que procuraram avaliar fatores associados ao bem-estar psicológico em adolescentes, identificando, na maioria da amostra estudada, expressões correspondentes aos dois maiores níveis de bem-estar. Além disso, é importante considerar o fato de que os participantes estavam freqüentando a escola, o que consiste em um fator protetivo nesta etapa do ciclo vital (Gallo \& Williams, 2008).

No caso do presente estudo, é importante considerar que os jovens eram estudantes de escolas públicas estaduais. Chaise, Soares e Meneghel (2008) encontraram diferença entre a percepção de saúde de adolescentes estudantes de escola pública e os de escola particular. Os estudantes de escola pública 
Tabela 2

Relação entre os domínios de satisfação com a vida, percepção de saúde e experiência de estados psicológicos (emoções) negativos em escolares da rede estadual de Cachoeirinha, Cachoeirinha, RS, 2008.

\begin{tabular}{lcccc}
\hline \multicolumn{1}{c}{ Satisfação } & $\begin{array}{c}\text { Percepção de } \\
\text { saúde }\end{array}$ & Deprimido/a & Nervoso/a & $\begin{array}{c}\text { Irritado/a ou } \\
\text { bravo/a }\end{array}$ \\
\hline Família & 0,033 & $-0,290^{* *}$ & $-0,171^{*}$ & $-0,167^{*}$ \\
Amigos & 0,031 & $-0,173^{*}$ & $-0,036$ & $-0,043$ \\
Experiência como estudante & $0,189^{*}$ & $-0,208^{* *}$ & $-0,251^{* *}$ & $-0,216^{* *}$ \\
Consigo mesmo & $0,160^{*}$ & $-0,340^{* *}$ & $-0,211^{* *}$ & $-0,164^{*}$ \\
Lugar onde vive & 0,054 & $-0,310^{* *}$ & $-0,182^{*}$ & $-0,170^{*}$ \\
Vida em geral & $0,156^{*}$ & $-0,300^{* *}$ & $-0,234^{* *}$ & $-0,122$ \\
\hline
\end{tabular}

$* \mathrm{p} \leq 0,05 ; * * \mathrm{p} \leq 0,01$

relacionaram a saúde com o bem-estar físico e mental, referindo aspectos como ser feliz, saúde, paz e alegria, enquanto que para os da instituição privada saúde foi apontada apenas como ausência de doenças.

Em relação à percepção de saúde, mais da metade dos participantes deste estudo $(67,1 \%)$ avaliouse como bastante ou muito saudável, frente a $2,7 \%$ que se avaliaram como nada saudáveis. Estes dados confirmam os achados de Garbin, Garbin, Moimaz e Gonçalves (2009), que verificaram que $46,5 \%$ dos adolescentes consideravam sua saúde ótima e 44,1\%, boa. Apenas $9 \%$ dos jovens consideraram sua saúde regular e $0,4 \%$ ruim.

Ao considerar as diferenças por sexo, este estudo identificou uma percepção de saúde mais negativa por parte das meninas, revelando diferença significativa entre os sexos. Estes dados corroboram os achados de pesquisas anteriores, como a de Galárraga, Aguilá e Rajmil (2009). Os autores identificaram clara diferença entre os sexos na percepção de saúde e na qualidade de vida relacionada à saúde, sendo que as meninas, principalmente as adolescentes, têm uma percepção pior de sua saúde geral, física e emocional, porém percebem como melhor sua relação com os amigos e no âmbito escolar. Afirmam ainda que poucos estudos investigam a diferença de gênero em relação à percepção de saúde, e os que o fizeram apontam que a partir da adolescência as meninas percebem sua saúde como pior que os meninos, porém de forma geral os adolescentes têm uma percepção positiva de saúde.

No que tange às diferenças entre os sexos em termos da satisfação com a vida, o presente estudo demonstrou diferenças entre os sexos nos domínios de satisfação com a vida, porém estas não chegaram a ser estatisticamente significativas. As meninas obtiveram médias maiores em cinco dos seis itens, apenas não no item de satisfação com o lugar em que vivem. Goldbeck, Schmitz, Besier, Herschbach e Henrich (2007), em estudo com 1.274 adolescentes alemães com idades entre 11 e 16 anos, encontraram índices significativamente mais baixos de satisfação com a vida em geral e com a saúde entre as meninas.
No entanto, estudo de Ma e Huebner (2008) sobre a satisfação de vida de adolescentes apresentou resultados semelhantes aos encontrados no presente estudo. Os autores encontraram que variáveis sóciodemográficas como classe socioeconômica e sexo eram fracos preditores da satisfação global de vida, enquanto as variáveis interpessoais constituíam preditores mais fortes.

Nesse sentido, buscou-se identificar como a satisfação com diversos domínios da vida dos adolescentes se relacionava à percepção de saúde, que foi significativamente mais positiva entre os meninos, e à experiência de sentir-se deprimido, nervoso e irritado ou bravo, que também se apresentou com médias significativamente superiores entre as meninas. Os resultados encontrados demonstraram relação entre os aspectos subjetivos da vida dos adolescentes (percepção de saúde e experiências psicológicas negativas) e os fatores sociais concretos (considerados a partir da satisfação dos adolescentes com os mesmos). Dados semelhantes foram apontados por Ozella e Aguiar (2008) que a partir do estudo realizado, afirmam que o sentido de ser adolescente é construído nas relações concretas e nas atividades significadas.

Uma percepção mais positiva de saúde está associada a uma maior satisfação em relação aos estudos, consigo mesmo, e com a vida em geral. Tais resultados permitem identificar que os aspectos relacionados às potencialidades pessoais, como ter maior satisfação com os estudos e consigo mesmo, são fatores primordiais para a percepção de uma saúde mais positiva. Conforme Traverso-Yépez e Pinheiro (2002), os adolescentes têm a necessidade de espaços apropriados para o desenvolvimento da auto-estima, da criatividade e de projetos de vida para conquistar uma maturidade saudável. Dentro do enfoque da promoção da saúde, o controle sobre os aspectos da própria vida conduz ao empoderamento dos indivíduos (Carvalho \& Gastaldo, 2008). Diaz e Alvarado (2007), em estudo com 927 trabalhadores e estudantes para avaliar o bem-estar subjetivo (seus 
componentes cognitivos e afetivos) e sua relação com variáveis sociodemográficas, também verificaram correlação positiva significativa entre percepção de saúde e satisfação. Os autores não encontraram diferenças entre os gêneros no que se refere à saúde global percebida e a saúde física especificamente, porém apontam diferenças na saúde mental, na qual as mulheres apresentam pior saúde que os homens.

Ao encontro desta afirmação, no presente estudo, os resultados relacionados à saúde mental (sentirse deprimido, nervoso e irritado), demonstraram diferenças estatisticamente significativas entre os sexos, sendo que as meninas apresentaram nos três itens, piores índices que os meninos. Uma maior freqüência em sentir-se deprimido e nervoso relacionou-se a menor satisfação com todos os domínios estudados. A experiência mais freqüente de sentir-se irritado ou bravo seguiu este mesmo padrão, apenas sem associar-se com a satisfação com a vida em geral.

Barros, Gropo, Petribú e Colares (2008) destacam que a satisfação com a vida é um dos aspectos que definem a qualidade de vida como um todo. O conceito de qualidade de vida é multidimensional, e inclui bem-estar (material, físico, social, emocional e produtivo) e satisfação em várias áreas da vida. As pesquisas que avaliam a qualidade de vida utilizam tanto indicadores objetivos quanto subjetivos, sendo estes dirigidos às estimativas subjetivas das circunstâncias de vida, como os julgamentos de satisfação e as emoções. É nesse sentido que uma avaliação negativa das relações que o adolescente estabelece em seu contexto contribui para um incremento do estresse vital que se manifesta através de nervosismo (ansiedade) e depressão, estados aos quais as meninas estão mais suscetíveis (Benincasa \& Rezende, 2006). De acordo com Reppold, Pacheco, Bardagi e Hutz (2002), os eventos estressantes geram um alto grau de tensão, interferindo nos padrões normais de resposta do indivíduo e associando-se a diversos distúrbios físicos e mentais. Já o sentir-se irritado ou bravo não se relaciona com a satisfação com a vida em geral, mas pode estar associado a uma resposta a ambientes hostis que podem predispor a conseqüências mal-adaptativas como a agressão (Holden, Geffner, \& Jouriles, 1998).

De acordo com Chaise, Soares e Meneghel (2008), foram criadas políticas públicas de saúde com o objetivo de promover a saúde, identificar as vulnerabilidades e reabilitar para os adolescentes, por estes apresentarem necessidades de saúde para além dos fatores orgânicos, porém esses programas ainda não alcançaram o objetivo de cuidar da integralidade dos adolescentes. Vê-se ainda uma prática voltada ao olhar clínico, e ao se falar em promoção e prevenção de saúde, fala-se de ações de controle, de medidas sanitárias com caráter autoritário e intervencionista, baseados no modelo médico focado na doença.
Percebe-se através dos resultados desta e das demais pesquisas citadas neste artigo que os dados apontam para a necessidade de se mudar o enfoque de risco e de vulnerabilidade que muitas vezes afeta os adolescentes, visto que não é desta forma que eles próprios percebem a sua saúde. Um redirecionamento do olhar dos profissionais que atuam junto a adolescentes é necessário para que seja possível rever os pré-conceitos relacionados a essa etapa da vida. Existe um grande potencial de saúde na adolescência e, sem dúvida, este é o ponto do qual devem partir as ações de promoção da saúde para esta população.

Como limitação deste estudo, ressalta-se o número pequeno da amostra. Nesse sentido, a pesquisa tem sido ampliada para outros municípios e escolas do Rio Grande do Sul, com vistas a verificar se os resultados se mantêm em outras realidades no sul do Brasil. Nesse sentido, sugere-se o desenvolvimento de outros estudos sobre o tema que pudessem contribuir para uma compreensão maior da saúde adolescente no Brasil, com base na percepção dos próprios.

\section{Referências}

Albuquerque, A. S., \& Tróccoli, B. T. (2004). Desenvolvimento de uma escala de bem-estar subjetivo. Psicologia: Teoria e Pesquisa, 20 (2), 153-164.

Barros, L. P., Gropo, L. N., Petribú, K., \& Colares, V. (2008). Avaliação da qualidade de vida em adolescentes - revisão da literatura. Jornal Brasileiro de Psiquiatria, 57 (3), 212-217.

Benincasa, M., \& Rezende, M. M. (2006). Tristeza e suicídio entre adolescentes: fatores de risco e proteção. Boletim de Psicologia, 55 (124), 93-110.

Carvalho, S. R., \& Gastaldo, D. (2008). Promoção à saúde e empoderamento: uma reflexão a partir das perspectivas críticosocial pós-estruturalista. Ciência e Saúde Coletiva,13 (supl.2), 2029-2040.

Chaise, F. O., Soares, S. A., \& Meneghel, S. N. (2008). Estão nossos jovens doentes? Percepções de adolescentes em relação à saúde em dois municípios gaúchos. Physis Revista de Saúde Coletiva, 18 (4), 817-828.

Costa, M. C. O., \& Bigras, M. (2007). Mecanismos pessoais e coletivos de proteção e promoção da qualidade de vida para a infância e adolescência. Ciência \& Saúde Coletiva, 12 (5), 11011109.

Cromack, L. M. F., Burstyn, I., \& Tura, L .F. R. (2009). O olhar do adolescente sobre saúde: um estudo de representações sociais. Ciência \& Saúde Coletiva, 14 (2), 627-634.

Diaz, E. M., \& Alvarado, N. R. (2007). Bienestar subjetivo: midiendo satisfacción vital, felicidad y salud en población chilena de la Región Maule. Revista Universum, 22 (2), 177-193.

Diener, E. (1994). El bienestar subjetivo. Intervención psicosocial. Revista sobre igualdad y calidad de vida, 3 (8), 67-113.

Ferreira, M. A., Alvinir, N. A. T., Teixeira, M. L. O., \& Veloso, R. C. (2007). Saberes de Adolescentes: estilo de vida e cuidado à saúde. Texto Contexto Enfermagem, 16 (2), 217-224.

Galárraga, R. V., Aguilá, S. L., \& Rajmil, L. (2009). Género y salud percibida em la infância y la adolescência em España. GacSanit, 23 (5), 433-439.

Gallo, A. E., \& Williams, L. C. A. (2008). A escola como fator de proteção à conduta infracional de adolescentes. Cadernos de Pesquisa, 38 (133), 41-59.

Garbin, C. A. S., Garbin, A. J. I., Moimaz, S. A. S., \& Gonçalves P. E. (2009). A saúde na percepção do adolescente. Physis Revista de Saúde Coletiva, 19 (1), 227-238. 
García, M. A. (2002). El bienestar subjetivo. Escritos de Psicología, 6, 18-39.

García-Viniegras, C., \& González, I (2000). La categoría bienestar psicológico, su relación con otras categorías sociales. Revista Cubana de Medicina Integral, 16 (6), 586-592.

Goldbeck, L., Schimitz, T. G., Besier, T. Herschbach, P. \& Henrich, G. (2007). Life satisfaction decreases during adolescence. Quality of Life Research, 16, 969-979.

Holden, G. W., Geffner, R., \& Jouriles, E. N. (1998). Children exposed to marital violence: theory, research, and applied issues. Washington: American Psychology Association.

Huebner, E.S., Suldo, S. M., Valois, R. R., Darne, J. W., \& Zullig, K. (2004). Brief Multidimensional Students' Life Satisfaction Scale: Sex, race, and grade effects for a high school sample. Psychological Reports, 94, 351-356.

Huici, C. (1985). Grupo social y comportamiento de salud y enfermedad. In F. J. Morales, F. J.(Eds.), Psicología Social Aplicada (pp. 111-130). Bilbao: Desclée de Brouwer.

Kuczynski, E. (2010). Qualidade de vida na infância e na adolescência. In Assumpção, F. B. Jr (Eds.), Qualidade de vida na infância e na adolescência: orientações para pediatras e profissionais da saúde mental (pp. 43-56). Porto Alegre: Artmed.

Ma, C. Q., \& Huebner, E. S. (2008). Attachment relationships and adolescents' life satisfaction: some relationships matter more to girls than boys. Psychology in the Schools, 45 (2), 177-190.

Ozella, S., \& Aguiar, W. M. J. (2008). Desmistificando a concepção de adolescência. Cadernos de Pesquisa, 38 (133), 97125.

Passareli, P. M., \& Silva, J. A. (2007). Psicologia Positiva e o estudo do bem-estar subjetivo. Estudos de Psicologia, 24 (4), 513517.

Reppold, C. T., Pacheco, J., Bardagi, M., \& Hutz, C. (2002). Prevenção de problemas de comportamento e desenvolvimento de competências psicossociais em crianças e adolescentes: uma análise das práticas educativas e dos estilos parentais. In Hutz, C. S. (Eds.), Situações de risco e vulnerabilidade na infância e na adolescência: aspectos teóricos e estratégias de intervenção (pp.
7-51). São Paulo: Casa do Psicólogo.

Rodríguez Marín, J. \& García, J. A. (1995). Estilo de vida y salud. In Latorre, J. M. (Ed.), Ciencias psicossociales aplicadas II (pp. 25-34). Madrid: Síntesis.

Rodríguez Marín, J., Martínez García, M. \& Valcárcel, P. (1990). Cirurgía y hospitalización como acontecimientos estresantes en pacientes oftalmológicos. Libro II Congreso del Colegio Oficial de Psicólogos, 5 (2), 117-122.

Secretaria da Educação do Rio Grande do Sul (2008). Escolas do Rio Grande do Sul. Acessado de http://www.educacao.rs.gov.br/ pse/html/educa.jsp.

Silva, R. A., Horta, B. L., Pontes, L. M., Faria, A. D., Souza, L. D. M., Cruzeiro, A. L, S., \& Pinheiro, R. T. (2007). Bem-estar psicológico e adolescência: fatores associados. Cadernos de Saúde Pública, 23 (5), 1113-1118

Traverso-Yépez, M., \& Pinheiro, V. S. (2002). Adolescência, saúde e contexto social: esclarecendo práticas. Psicologia \& Sociedade, 14 (2), 133-147.

Veenhoven, R. (1994). El estudio de la satisfacción con la vida. Intervención Psicosocial, 3, 87-116.

Wold, B. (1995). Health behavior in schoolchildren: A WHO cross-national Surrey. Resource Package Questions 1993-94. Norway: University of Bergen.

Sobre os autores:

Miriam Raquel Walchholz Strelhow - Graduanda em Psicologia (ULBRA/Canoas); bolsista $\mathrm{IC} / \mathrm{CNPQ}$.

E-mail: raquelwch@gmail.com

Cheila de Oliveira Bueno - Psicóloga (ULBRA/Canoas).

Sheila Gonçalves Câmara - Psicóloga; Doutora em Psicologia; Docente do Curso de Psicologia e do Programa de Pós-Graduação em Saúde Coletiva (ULBRA/Canoas e do Departamento de Psicologia da Universidade Federal de Ciências da Saúde de Porto Alegre. 